Reprod. Nutr. Dévelop., 1987, 27 (1 B), 225-226.

\title{
Evaluation de la technique du double marquage appliquée à la mesure du flux duodénal chez le mouton
}

C. PONCET, A. AL ABD

Laboratoire de la Digestion des Ruminants, I.N.R.A. Theix, 63122 Ceyrat, France.

Summary. The validity of the double marker technique for duodenal flow has been tested using different markers in sheep fitted with abomasal and duodenal simple cannulas. The compositions of abomasal and duodenal contents after correction were no longer significantly different. The corrected flows were less subject to marker and sampling site variations.

La technique du double marquage (Faichney, 1980) est très diversement appréciée des utilisateurs, en raison de l'imperfection des marqueurs, mais les études critiques sont rares, en l'absence de valeurs de référence. La validité de la correction par double marquage, effectuée avec les principaux types de marqueurs, a été estimée en considérant son effet sur la dispersion des valeurs de flux et par la convergence des compositions et des flux corrigés des contenus digestifs de la caillette et du duodénum prélevés simultanément sur le même animal.

Matériel et méthodes. Quatre moutons adultes munis d'une canule du rumen et d'une canule simple de la caillette et du duodénum ont reçu 2 rations $(900 \mathrm{~g}$ de matière organique $(\mathrm{MO})$ en 8 repas égaux/jour): I'une à base de foin haché $(80 \%)$, l'autre à base d'aliment concentré $(65 \%)$ et de foin $(35 \%)$. Les marqueurs $-\mathrm{Cr}_{2} \mathrm{O}_{3}$ sur support cellulose $\left(\mathrm{Cr}\right.$ cell) et $\mathrm{Cr}_{2} \mathrm{O}_{3}$ fixé par mordançage $(\mathrm{Cr}$ mord) - ont été distribués à chaque repas ; l'ytterbium ( $\left.{ }^{169} \mathrm{Yb}\right)$ en solution a été instillé en continu par la canule du rumen comme les marqueurs de la phase liquide : $P E G$ et ${ }^{51} \mathrm{Cr}$-EDTA. Douze prélèvements effectués simultanément au niveau de la caillette (C) et du duodénum (D) pendant 3 jours consécutifs ont été traités selon la technique du DM (filtration) pour constituer 2 échantillons moyens par animal, contenu total et filtrat, sur lesquels ont été déterminées les teneurs en marqueurs, MO et azote (N). Les flux de MO et de $\mathrm{N}$ ont été calculés aux 2 niveaux $C$ et $D$ par simple marquage avec chacun des 5 marqueurs et par DM associant $\mathrm{Cr}$-mord/PEG, $\mathrm{Cr}$-cell/PEG et ${ }^{169} \mathrm{Yb} /{ }^{51} \mathrm{Cr}$-EDTA.

Résultats et discussion. Pour les 2 rations étudiées, les teneurs en $\mathrm{MO}$ des contenus $\mathrm{C}$ ont été supérieures, de $11 \%(\mathrm{P}<5 \%)$, à celles des contenus $D: 54,5$ contre $49,1 \%$. Après correction par DM, l'écart entre les teneurs en MO est réduit de moitié $(45,9$ et $43,4 \%$ respectivement pour $C$ et $D$ ) et l'effet du lieu de prélèvement disparaît. En rapprochant les compositions des contenus digestifs $C$ et $D$, qui devraient être identiques après correction, le DM a l'effet positif attendu; mais la correction est incomplète, surtout avec le couple $\mathrm{Cr}$ cell/PEG.

La dispersion des flux de MO (\% MO Ingérée, MOI) et d'N (/NI) calculés avec chacun des 5 marqueurs est élevée, surtout au niveau de la caillette (tabl. 1). Les flux de $\mathrm{MO}$ estimés par simple marquage à partir des échantillons $\mathrm{C}$ sont supérieurs $(P<1 \%$ ) à ceux calculés à partir des échantillons $D(62,4$ contre 
$59,3 \% \mathrm{MOI})$. Les marqueurs de la phase liquide donnent des flux supérieurs $(\mathrm{P}<1 \%$ ) à ceux calculés avec les marqueurs de la phase solide, et les flux calculés avec le $\mathrm{Cr}$-mord. sont inférieurs $(P<1 \%$ à ceux obtenus avec $\mathrm{Cr}$-cell et ${ }^{169} \mathrm{Yb}$, qui sont quasiment identiques. Le DM réduit considérablement cette dispersion liée au choix du marqueur : la correction des flux $\mathrm{Cr}$-mord est importante, bien qu'insuffisante; celle apportée aux flux $\mathrm{Cr}$-cell et $\mathrm{Yb}$ est relativement faible et non significative, de sorte que les flux DM ne sont pas significativement différents entre eux. Le DM réduit également l'écart entre les flux $C$ et $D(57,7$ et $56,4 \%$ MOl en moyenne) qui n'est plus significatif mais demeure cependant plus grand avec le couple $\mathrm{Cr}$-cell/PEG. Les mêmes observations peuvent être faites pour les flux d'azote.

TABL. 1. - Influence du marqueur de la phase solide - Cr-mord (1), Cr-cell (2), ${ }^{169}$ Yb (3) - , et de la phase liquide - PEG (4), ${ }^{51} \mathrm{Cr}$-EDTA (5) - et du mode de calcul - simple marquage (1 à 5 ) et double marquage $(1+4,2+4,3+5)$ - sur les flux duodénaux de MO (\% de la MO ingérée : MOl) et de $N$ (rapporté à $\mathrm{N}$ ingéré : /NI) mesurées par prélèvements dans la caillette (C) et dans le duodénum (D), dans le cas de 2 rations à base de foin $(80 \%)$ ou d'aliments concentrés $(65 \%)$ distribués à 4 moutons en 8 repas/jour ( $\sigma=$ moyenne des écart-types).

\begin{tabular}{|c|c|c|c|c|c|c|c|c|c|c|c|c|}
\hline & & 1 & 2 & 3 & $\bar{\sigma}$ & 4 & 5 & $\bar{\sigma}$ & $1+4$ & $2+4$ & $3+5$ & $\bar{\sigma}$ \\
\hline \multicolumn{13}{|l|}{ Ration " Foin " } \\
\hline \multirow[t]{2}{*}{ - Flux MO (\%MOI) } & C & 50,5 & 64,7 & 60,7 & 4,0 & 70,2 & 63,7 & 8,2 & 54,2 & 62,3 & 58,5 & 5,9 \\
\hline & 5 & 54,9 & 60,9 & 60,3 & 3,6 & 67,6 & 62,4 & 5,1 & 56,6 & 58,9 & 58,6 & 4,7 \\
\hline \multirow[t]{2}{*}{- Flux $\mathrm{N} \quad(/ \mathrm{NI})$} & $\mathrm{C}$ & 0,95 & 1,20 & 1,13 & 0,07 & 1,29 & 1,18 & 0,08 & 1,16 & 1,21 & 1,14 & 0,06 \\
\hline & D & 1,10 & 1,15 & 1,14 & 0,06 & 1,30 & 1,18 & 0,07 & 1,23 & 1,20 & 1,15 & 0,05 \\
\hline \multicolumn{13}{|l|}{$\begin{array}{l}\text { Ration "Concen- } \\
\text { trés " }\end{array}$} \\
\hline \multirow[t]{2}{*}{ - Flux MO (\%MOI) } & C & 49,1 & 60,1 & 63,0 & 5,8 & 71,7 & 70,6 & 12,5 & 53,3 & 58,2 & 59,6 & 6,5 \\
\hline & $D$ & 51,3 & 56,0 & 56,2 & 3,9 & 62,0 & 61,2 & 13,2 & 53,5 & 55,5 & 55,5 & 5,1 \\
\hline \multirow[t]{2}{*}{- Flux N (/NI) } & C & 0,68 & 0,90 & 0,93 & 0,06 & 1,04 & 1,04 & 0,18 & 0,95 & 0,95 & 0,96 & 0,07 \\
\hline & D & 0,87 & 0,90 & 1,01 & 0,10 & 1,02 & 1,10 & 0,21 & 1,14 & 0,90 & 1,02 & 0,05 \\
\hline \multicolumn{4}{|l|}{$\begin{array}{l}\text { Effets " marqueurs" } \\
\text { ou " DM " } \\
\text { lieu de prélèvement }\end{array}$} & \multicolumn{4}{|c|}{$\begin{array}{l}\mathbf{S}(P<1 \%) \\
\mathrm{S}(\mathrm{P}<1 \% \%)\end{array}$} & & & & $\begin{array}{l}\text { NS } \\
\text { NS }\end{array}$ & \\
\hline
\end{tabular}

Ainsi, le DM améliore la qualité des mesures de flux digestif en réduisant les différences importantes observées entre les flux calculés par simple marquage. Sa validité est surtout confirmée par le rapprochement des flux $C$ et $D$. Le DM $\mathrm{Yb} / \mathrm{CrEDTA}$ a conduit à des résultats généralement plus satisfaisants que les autres couples de marqueurs, bien que le couple $\mathrm{Cr}$-cell/PEG classiquement utilisé conduise à des valeurs souvent très proches. Enfin, les flux de $\mathrm{MO}$ et de $\mathrm{N}$ calculés avec $\mathrm{Yb}$ et $\mathrm{Cr}$-cell utilisés seuls ou en DM diffèrent peu entre eux, surtout au niveau duodénal. 